EPJ Web of Conferences 21, 04002 (2012)

DOI: $10.1051 /$ epjconf/20122104002

(c) Owned by the authors, published by EDP Sciences, 2012

\title{
Dynamical phase transitions in quantum mechanics
}

\author{
Ingrid Rotter ${ }^{\mathrm{a}}$ \\ Max Planck Institute for the Physics of Complex Systems, D-01187 Dresden, Germany
}

\begin{abstract}
The nucleus is described as an open many-body quantum system with a nonHermitian Hamilton operator the eigenvalues of which are complex, in general. The eigenvalues may cross in the complex plane (exceptional points), the phases of the eigenfunctions are not rigid in approaching the crossing points and the widths bifurcate. By varying only one parameter, the eigenvalue trajectories usually avoid crossing and width bifurcation occurs at the critical value of avoided crossing. An analog spectroscopic redistribution takes place for discrete states below the particle decay threshold. By this means, a dynamical phase transition occurs in the many-level system starting at a critical value of the level density. Hence the properties of the low-lying nuclear states (described well by the shell model) and those of highly excited nuclear states (described by random ensembles) differ fundamentally from one another. The statement of Niels Bohr on the collective features of compound nucleus states at high level density is therefore not in contradiction to the shell-model description of nuclear (and atomic) states at low level density. Dynamical phase transitions are observed experimentally in different quantum mechanical systems by varying one or two parameters.
\end{abstract}

\section{Introduction}

In 1936, Niels Bohr wrote in the address delivered on January 27 before the Copenhagen Academy [1]: In the atom and in the nucleus we have indeed to do with two extreme cases of mechanical many-body problems for which a procedure of approximation resting on a combination of one-body problems, so effective in the former case, loses any validity in the latter where we, from the very beginning, have to do with essential collective aspects of the interplay between the constituent particles. At that time, narrow compound nucleus states at high excitation energy and shell model states in atoms at low excitation energy were known.

About 20 years later, it could be shown that the spectra of nuclei at low excitation energy are described well on the basis of the shell model [2], i.e. on the basis of an approximation resting on a combination of one-body problems. The shell closures in nuclei differ from those in atoms since the symmetries of nuclear forces differ from those of the forces in atoms. The individual nuclear states at low level density are described, in the nuclear shell model, by using two-body residual forces. The finite lifetimes of nuclear states are calculated, usually, perturbatively, i.e. the particle decay is assumed to take place by tunneling of the particle through a barrier.

It is difficult to find a convincing answer to the question Can the compound nucleus be described by the shell model for the following reasons.

(i) Due to the strong nuclear forces, there is (at present) no parameter that allows to control the behavior of the nuclear states as a function of its variation. It is possible only to control average values as a function of energy.

(ii) By means of different sets of fitting parameters, different interpretations of the experimental data are usually possible. An example are the problems arising in the identification of doorway states by using standard methods [3].

\footnotetext{
a e-mail: rotter@pks.mpg.de
}

This is an Open Access article distributed under the terms of the Creative Commons Attribution-Noncommercial License 3.0, which permits unrestricted use, distribution, and reproduction in any noncommercial medium, provided the original work is properly cited. 


\section{EPJ Web of Conferences}

(iii) The nuclear forces are residual forces that are not derived from first principles but are described by means of parameters that are fitted to many nuclei.

Also the question on a possible non-Hermitian contribution to the Hamilton operator describing nuclear states, is not answered clearly in the theoretical studies. In the shell model, the Hamilton operator describing nuclear states at low excitation energy, is assumed to be Hermitian. The finite lifetimes of the states (decay widths) are calculated perturbatively, and the feedback from the continuum onto the shell model states is not at all taken into account in the concrete calculations.

The compound nucleus states are described usually by means of random ensembles, where the question of the Hermiticity or non-Hermiticity of the Hamiltonian is avoided. In these studies, average lifetimes are considered instead of the lifetimes of individual states. Also the question of a feedback from the continuum onto the system is not considered.

Over the years, some problems occurred in describing experimental results on nuclei by means of the standard statistical theory. Here, only two examples will be mentioned.

(i) The mean compound nucleus lifetime increases, experimentally, with increasing excitation energy in the regime of overlapping resonances. This result has been found many years ago for the mean compound-nucleus lifetime in proton induced reactions on $\mathrm{Ni}$ isotopes by using the crystal blocking technique [4]. The mean lifetime is determined at the energies $E=5.65$ and $6.50 \mathrm{MeV}$. It is significantly longer at the higher bombarding energy than at the lower one, contrary to expectations of a purely statistical theory, and is much larger than expected. Furthermore, the data suggest an interpretation in terms of some form of intermediate structure resulting from the local spreading of a comparatively simple configuration [4]. Unfortunately, the experimental studies are not continued because of the direct contradiction of the results to the basic assumptions of the statistical theory [5]. Today we know that the picture obtained experimentally fits in with the spectroscopic redistribution processes taking place in the regime of overlapping resonances under the influence of the environment $[6,7]$.

(ii) The reduced neutron widths of compound nucleus states do, experimentally, not follow the PorterThomas distribution as has been shown recently [8].

There are also theoretical problems in the relation between compound nucleus states, described mostly by the Gaussian orthogonal ensemble (GOE), and shell model states. The first problem is that the GOE contains many-body forces, i.e. collective features. It can not be related to a two-body random ensemble [9]. Further, the states of the GOE do not decay according to an exponential law in the fewchannel case [10]. That means, they cannot be considered to correspond to isolated states although they are well separated from one another in energy. Further, the coupling matrix elements between bound and scattering states calculated for ${ }^{24} \mathrm{Mg}$ on the basis of the continuum shell model, do not agree with the assumption of the GOE on channel equivalence and vanishing real part [11].

According to these problems, it is necessary to reconsider the shell model description of nuclear states, by starting from the scenario at low level density and continuing to that at high level density. Most interesting is the regime of overlapping resonances where spectroscopic redistribution processes are known to occur.

In section 2, the Feshbach projection operator formalism is sketched on the basis of which the present study is performed. In this formalism, the non-Hermitian operator $H_{\text {eff }}$ describes the coupling of the system to the environment into which it is embedded. The non-trivial mathematical features of non-Hermitian operators are illustrated in section 3 by means of a $2 \times 2$ operator describing a two-level system. In the following section 4 , the role of non-Hermitian operators in the full manyparticle system is considered. Spectroscopic redistribution processes are shown to occur under the influence of the coupling to the environment. Width bifurcation in the two-level system causes, at high level density, the formation of a few aligned short-lived states together with trapped long-lived states. Mathematically, the spectroscopic redistribution is possible due to the nonrigid phases of the wavefunctions. It causes a dynamical phase transition at high level density, section 5. Examples of dynamical phase transitions observed in different experimental data are sketched in section 6 . The results are summarized in the last section 7 and related to Bohr's statement on the differences between shell model and compound nucleus states. 
$\mathrm{CNR} * 11$

\section{Feshbach projection operator technique with a non-Hermitian effective Hamilton operator}

In an open quantum system the states have a finite lifetime due to their coupling to the environment of scattering states. In order to describe it, the concepts system and environment have to be defined clearly.

In the following, the system considered is a many-particle system with many levels. It is described by standard quantum mechanics (e.g. the nuclear shell model) and is localized in space. The (natural) environment of the system is the continuum of scattering wavefunctions into which the system is embedded. Also the environment is described by standard quantum mechanics (scattering theory). In contrast to the system, it is extended in space. Due to embedding the system into the environment, the states of the system have, generally, a finite lifetime. In order to allow for a possible feedback between system and environment, the coupling between system and environment has, generally, to be taken into account nonperturbatively.

Let us sketch the Feshbach projection operator formalism [12] that allows a unified description of structure and reaction, for details see [7]. The structure is determined by the spectroscopic properties of the system ( $Q$ subspace) while the reaction is induced by the environment of scattering wavefunctions ( $P$ subspace).

The Schrödinger equation in the total function space reads

$$
\left(H^{\text {full }}-E\right) \Psi_{c}^{E}=0
$$

where

$$
H^{\text {full }} \equiv H_{Q Q}+H_{Q P}+H_{P Q}+H_{P P}
$$

is Hermitian and $H_{Q Q} \equiv Q H Q, H_{Q P} \equiv Q H P$ and so on. The two projection operators $Q$ and $P$ are defined by

$$
\begin{aligned}
& \left(H_{B}-E_{k}^{B}\right) \Phi_{k}^{B}=0 \longrightarrow Q=\sum_{k=1}^{N}\left|\Phi_{k}^{B}\right\rangle\left\langle\Phi_{k}^{B}\right| \\
& \left(H_{c}-E\right) \xi_{c}^{E}=0 \quad \longrightarrow P=\sum_{c=1}^{C} \int_{\epsilon_{c}}^{\epsilon_{c}^{\prime}} d E\left|\xi_{c}^{E}\right\rangle\left\langle\xi_{c}^{E}\right|
\end{aligned}
$$

and $P+Q=1$ is assumed. The operator $H_{B}=H_{B}^{0}+V$ describes the discrete states of the system with energies $E_{k}^{B}$ and wavefunctions $\Phi_{k}^{B}$. It contains the two-body residual interaction $V$ between the particles. The operator $H_{c}$ describes the continuum with wavefunctions $\xi_{c}^{E}$ at the energy $E$. The coupling between the discrete and scattering states is given by

$$
\hat{\gamma}_{k}^{c}=\sqrt{2 \pi}\left\langle\xi_{c}^{E}|V| \Phi_{k}^{B}\right\rangle .
$$

Thus, $\Psi_{c}^{E}$ contains (by definition) everything and $H^{\text {full }}$ is Hermitian. The solution of the Schrödinger equation in the total function space reads [7]

$$
\Psi_{c}^{E}=\xi_{c}^{E}+\sum_{k, l=1}^{N}\left(\Phi_{k}^{B}+\omega_{k}^{0}\right)\left\langle\Phi_{k}^{B}\left|\frac{1}{E-H_{\mathrm{eff}}}\right| \Phi_{l}^{B}\right\rangle\left\langle\Phi_{l}^{B}\left|H_{Q P}\right| \xi_{c}^{E}\right\rangle
$$

where

$$
H_{\mathrm{eff}}=H_{B}+V_{B C} \frac{1}{E^{+}-H_{C}} V_{C B}
$$

is a non-Hermitian operator, and

$$
\omega_{k}^{0} \equiv G_{P}^{(+)} H_{P Q} \cdot \Phi_{k}^{B} ; \quad G_{P}^{(+)}=P\left(E-H_{P P}\right)^{-1} P .
$$




\section{EPJ Web of Conferences}

The solution (6) is called formal solution by Feshbach.

The operator $H_{\text {eff }}$ consists of a first-order and a second-order interaction term with

$$
\begin{aligned}
& \operatorname{Re}\left\{\left\langle\Phi_{i}^{B}\left|H_{\mathrm{eff}}\right| \Phi_{j}^{B}\right\rangle\right\}=\left\langle\Phi_{i}^{B}\left|H_{B}\right| \Phi_{j}^{B}\right\rangle+\frac{1}{2 \pi} \sum_{c} \mathcal{P} \int_{E_{\mathrm{thr}}^{l}}^{E_{\mathrm{thr}}^{h}} \mathrm{~d} E^{\prime} \frac{\hat{\gamma}_{i}^{c} \hat{\gamma}_{j}^{c}}{E-E^{\prime}} \\
& \operatorname{Im}\left\{\left\langle\Phi_{i}^{B}\left|H_{\mathrm{eff}}\right| \Phi_{j}^{B}\right\rangle\right\}=-\frac{1}{2} \sum_{c} \hat{\gamma}_{i}^{c} \hat{\gamma}_{j}^{c} .
\end{aligned}
$$

Here, $\mathcal{P}$ denotes the principal value integral, (10) is the residuum and $E_{\mathrm{thr}}^{h} \rightarrow \infty$ for nuclei. The direct (first-order) interaction $V$ is included in $H_{B}$ and its eigenfunctions $\Phi_{k}^{B}$.

After diagonalizing $H_{\mathrm{eff}}$,

$$
\left(H_{\mathrm{eff}}-z_{k}\right) \Phi_{k}=0
$$

with the eigenvalues $z_{k}$ and eigenfunctions $\Phi_{k}$, the solution (6) reads

$$
\Psi_{c}^{E}=\xi_{c}^{E}+\sum_{k=1}^{N} \Omega_{k} \cdot \frac{\left\langle\Phi_{k}^{*}\left|H_{Q P}\right| \xi_{c}^{E}\right\rangle}{E-z_{k}} .
$$

Here

$$
\Omega_{k}=\left(1+G_{P}^{(+)} H_{P Q}\right) \Phi_{k} \equiv\left(1+\omega_{k}\right) \Phi_{k}
$$

is the wavefunction of the resonance state $k$. The tail of the resonance wavefunction is determined by $\omega_{k}$, i.e. by a value being analog to (8).

The solution (12) is exact in relation to the assumption $P+Q=1$. A concrete proof is performed for potential scattering in the single-channel case [13].

It should be underlined here that the non-Hermitian Hamilton operator $H_{\text {eff }}$ appears in an intermediate stage of the formalism. It describes the subsystem that is localized in the $Q$ subspace and is coupled to the common environment of scattering wavefunctions ( $P$ subspace). The Hamiltonian $H^{\text {full }}$ corresponding to the total function space $Q+P=1$ is, of course, Hermitian.

The eigenvalues

$$
z_{k}=E_{k}-\frac{i}{2} \Gamma_{k}
$$

of $H_{\mathrm{eff}}$ are complex, in general, and the eigenfunctions $\Phi_{k}$ are complex and biorthogonal,

$$
\left\langle\Phi_{k}^{*} \mid \Phi_{k^{\prime}}\right\rangle=\delta_{k, k^{\prime}},
$$

such that

$$
\left\langle\Phi_{k} \mid \Phi_{k}\right\rangle \equiv A_{k} \geq 1
$$

The eigenvalues $z_{k}$ and eigenfunctions $\Phi_{k}$ are energy dependent functions due to the explicit energy dependence of $H_{\text {eff }}$.

The role of boundary conditions can be seen in the following manner. Inside the energy window coupled directly to the environment $\left(E_{\mathrm{thr}}^{l} \leq E \leq E_{\mathrm{thr}}^{h}\right)$, the states of the system are resonance states. Here, $H_{\mathrm{eff}}$ is non-Hermitian, the $z_{k}$ are complex, in general, the $\Phi_{k}$ are complex and biorthogonal, in general, and $\left\langle\Phi_{k}^{*} \mid \Phi_{k^{\prime}}\right\rangle=\delta_{k, k^{\prime}}$ according to (15).

Beyond the energy window, however, the states remain discrete. Here, $H_{\mathrm{eff}}$ is Hermitian, the $z_{k}=$ $E_{k}$ are real, i.e. $\Gamma_{k}=0$, but $E_{k} \neq E_{k}^{B}$ due to the principal value integral in (9). The $\Phi_{k}$ are orthogonal, $\left\langle\Phi_{k} \mid \Phi_{k^{\prime}}\right\rangle=\delta_{k, k^{\prime}}$. It should be underlined that the Hamiltonian $H_{\text {eff }}$ describes the states inside the energy window $E_{\mathrm{thr}}^{l} \leq E \leq E_{\mathrm{thr}}^{h}$ as well as beyond it. 


\section{$\mathrm{CNR} * 11$}

Of special interest is the solution of (1) inside the (localized) system. According to (12) and (13), it can be represented in a set $\left\{\Phi_{k}\right\}$ of biorthogonal wavefunctions,

$$
\left|\Psi_{c \text { int }}^{E, R}\right\rangle=\sum_{k=1}^{N} c_{k E}\left|\Phi_{k}\right\rangle ; \quad\left\langle\Psi_{c \text { int }}^{E, L}\right|=\sum_{k=1}^{N} c_{k E}\left\langle\Phi_{k}^{*}\right|
$$

with the coefficients

$$
c_{k E}=\frac{\left\langle\Phi_{k}^{*}\left|H_{Q P}\right| \xi_{c}^{E}\right\rangle}{E-z_{k}} \equiv \frac{1}{\sqrt{2 \pi}} \frac{\gamma_{k}^{c}}{E-z_{k}}
$$

which depend on energy.

\section{Biorthogonal wavefunctions, exceptional points and width bifurcation in the case of a two-level system}

\subsection{Exceptional points, true and avoided crossings}

Many years ago, Kato [14] introduced the notation exceptional points for singularities appearing in the perturbation theory for linear operators. Consider a family of operators of the form

$$
T(\varkappa)=T(0)+\varkappa T^{\prime}
$$

where $x$ is a scalar parameter, $T(0)$ is the unperturbed operator and $\chi T^{\prime}$ is the perturbation. Then the number of eigenvalues of $T(x)$ is independent of $x$ with the exception of some special values of $x$ where (at least) two eigenvalues coalesce. These special values of $\varkappa$ are the exceptional points. An example is the operator

$$
T(\varkappa)=\left(\begin{array}{cc}
1 & \varkappa \\
\varkappa & -1
\end{array}\right) .
$$

In this case, the two values $\varkappa= \pm \mathrm{i}$ give the same eigenvalue 0 .

Operators of the type (20) appear in the description of physical systems, for example in the theory of open quantum systems [7]. In this case, they represent a $2 \times 2$ Hamiltonian describing a two-level system with the unperturbed energies $\epsilon_{1}$ and $\epsilon_{2}$ and the interaction $\omega$ between the two levels,

$$
H(\omega)=\left(\begin{array}{ll}
\epsilon_{1} & \omega \\
\omega & \epsilon_{2}
\end{array}\right)
$$

The $\epsilon_{k}(k=1,2)$ and $\omega$ are complex usually, and $\operatorname{Re}\left(\epsilon_{k}\right)$ is the energy of the state $k$ while $2 \operatorname{Im}\left(\epsilon_{k}\right)$ is its decay width (inverse lifetime). In an open quantum system, two states can interact directly as well as via an environment [7]. In the present paper, we consider the case that the direct interaction is contained in the energies $\epsilon_{k}$ (see section 2). Then $\omega$ contains exclusively the coupling of the states via the environment which, in the case of an open quantum system, consists of the continuum of scattering wavefunctions into which the system is embedded. This allows to study environmentally induced effects in open quantum systems in a very clear manner.

The eigenvalues of the operator $H(\omega)$ are

$$
\varepsilon_{1,2}=\frac{\epsilon_{1}+\epsilon_{2}}{2} \pm Z ; \quad Z=\frac{1}{2} \sqrt{\left(\epsilon_{1}-\epsilon_{2}\right)^{2}+4 \omega^{2}} .
$$

The levels repel each other in energy according to the value $\operatorname{Re}(Z)$ while the widths bifurcate corresponding to $\operatorname{Im}(Z)$. The two eigenvalue trajectories cross when $Z=0$, i.e. when

$$
\frac{\epsilon_{1}-\epsilon_{2}}{2 \omega}= \pm i
$$




\section{EPJ Web of Conferences}

At these crossing points, the two eigenvalues coalesce,

$$
\varepsilon_{1}=\varepsilon_{2} \equiv \varepsilon_{0} \text {. }
$$

The crossing points may be called therefore exceptional points. In the vicinity of the crossing point, the dependence of the eigenvalue trajectories on the parameter is more complicated than far from them: the two levels approach each other in energy and the widths become equal so that $\operatorname{Re}\left(\varepsilon_{1}\right) \leftrightarrow \operatorname{Re}\left(\varepsilon_{2}\right)$ and $\operatorname{Im}\left(\varepsilon_{1}\right) \leftrightarrow \operatorname{Im}\left(\varepsilon_{2}\right)$ at the crossing point. In order to find the crossing point, it is necessary usually to vary two parameters. As a function of only one parameter, the states avoid crossing at the critical value of the parameter, in general.

The exceptional (crossing) points are points in the continuum of scattering wavefunctions (which represents the environment). They are therefore of measure 0 and can not be observed directly. However, they influence the behavior of the eigenvalue trajectories $\varepsilon_{k}(\alpha)$ (where $\alpha$ is a certain parameter) in their neighborhood in a non-negligible manner. Thus, the most interesting features of the exceptional (crossing) points in physical systems are their effects onto the eigenvalue trajectories $\varepsilon_{k}(\alpha)$ in a finite parameter range around the critical value $\alpha=\alpha_{\mathrm{cr}}$ and, furthermore, the behavior of the eigenvalue trajectories in approaching the crossing point, $\varepsilon_{k}(\alpha) \rightarrow \varepsilon_{k}\left(\alpha_{\mathrm{cr}}\right)$. An exceptional point defines a threshold for spectroscopic redistribution (caused by width bifurcation).

\subsection{The eigenvalues of a non-Hermitian $2 \times 2$ Hamilton operator}

We consider the Hamiltonian (21) with the unperturbed energies $\epsilon_{i}(i=1,2)$ of the two states and the interaction $\omega$ between them. The interaction $\omega$ contains exclusively the coupling of the states via the environment, which consists of the continuum of decay channels into which the states are embedded. The interaction $\omega$ is therefore a second-order interaction term. The two eigenvalues $\varepsilon_{k}(k=1,2)$ of (21) are given in (22). The Hamiltonian may be Hermitian or non-Hermitian.

For a Hermitian operator, the unperturbed energies $\epsilon_{i}$ of the states are real. The interaction $\omega$ being the principal value integral of the coupling term via the continuum, is also real [7]. Accordingly, the two eigenvalue trajectories $\varepsilon_{i}(\alpha)=e_{i}(\alpha)$ (where $e_{i}(\alpha)$ is real) cannot cross (for $\omega \neq 0$ ) when traced as a function of a certain parameter $\alpha$, see (22). Instead, they avoid crossing. This phenomenon is very well known for about 70 years [15]. The fictive crossing point is called usually diabolic point. The topological structure of this point is characterized by the Berry phase [16] which is studied theoretically and experimentally in many papers. The phenomenon of avoided crossing of discrete states can be traced back to the existence of a crossing (exceptional) point by analytical continuation into the continuum $[7,17]$.

The situation is another one for a non-Hermitian operator. In such a case, the unperturbed energies $\epsilon_{i}$ are usually complex. Also the interaction $\omega$ is complex, in general, since it contains the principal value integral as well as the residuum of the coupling term describing the interaction of the two states via the environment, see (9) and (10). The states can decay, in general, and the two eigenvalues of (21) can be written as

$$
\varepsilon_{1,2}=e_{1,2}-\frac{i}{2} \gamma_{1,2} \quad\left(\text { with } \gamma_{1,2} \geq 0\right)
$$

The widths $\gamma_{i}$ are proportional to the inverse lifetimes $\tau_{i}^{-1}$ of the states, $i=1,2$, see e.g. [18]. The two eigenvalue trajectories $\varepsilon_{i}(\alpha)$ may cross according to (22) and (24). The crossing point is an exceptional point, see section 3.1. The topological phase of the exceptional point is twice the Berry phase [7,19]. This theoretical result is proven experimentally by means of a microwave cavity [20].

According to the eigenvalue equation (22), $Z$ is complex usually. $\operatorname{Re}(Z)$ causes repulsion of the levels in energy. It is the dominant part when the resonance states are narrow (long-lived), i.e. when the interaction $|\omega|$ of the states via the continuum of scattering wavefunctions is small. The value $\operatorname{Im}(Z)$ has another physical meaning. It is the dominant part when $|\omega|$ is large what is the case, above all, when the two resonances overlap. According to $(22), \operatorname{Im}(Z)$ is related to a bifurcation of the widths of the levels. 


\section{$\mathrm{CNR} * 11$}

Due to width bifurcation, resonance states with long lifetime may appear together with short-lived states. The time scales characterizing these two different types of states, may differ strongly from one another. It is possible even that the widths of some states vanish, i.e. that $\gamma_{i}=0$ for some states. These states with vanishing width are called, usually, bound states in the continuum [21]. Examples are studied, e.g., in calculations for laser-induced continuum structures in atoms [22] as well as for the transmission through quantum dots [23]. In these calculations, resonance states with zero width appear at realistic parameter values. Tracing their appearance as a function of a parameter, one can see that they are nothing but special resonance states. The only hint in the cross section to such a state is the (elastic) scattering phase shift which passes into a jump by $\pi$ at the energy of the state, see Fig. 5 in [23] for an example. These bound states in the continuum coexist with short-lived states. In [24, $25]$, the bound states in the continuum are called spectral singularities.

Hence, the real and imaginary parts of the complex eigenvalues (25) of the Hamiltonian (21) have a physical meaning in an open quantum system in which the localized states of the system are embedded in an extended continuum of scattering states. The real parts $e_{i}$ stand for the positions in energy of the (almost) localized states while the imaginary parts $\gamma_{i}$ give the widths (inverse lifetimes) of these states. It is $\gamma_{i}>0$ when the decay of the states is not forbidden by any selection rule (and when the states are inside the energy window coupled to the continuum). Only at strong coupling to the continuum [corresponding to $\operatorname{Im}(Z) \gg \operatorname{Re}(Z)$ in $(22)$ ], discrete states may appear (due to width bifurcation) also inside the energy window coupled to the continuum.

\subsection{The eigenfunctions of a non-Hermitian $2 \times 2$ Hamilton operator}

The eigenfunctions of the non-Hermitian Hamilton operator $H$ are biorthogonal,

$$
\left\langle\phi_{k}^{*} \mid \phi_{l}\right\rangle=\delta_{k, l} \text {. }
$$

From these equations follows

$$
\left\langle\phi_{k} \mid \phi_{k}\right\rangle \equiv A_{k} \geq 1
$$

and

$$
\left\langle\phi_{k} \mid \phi_{l \neq k}\right\rangle=-\left\langle\phi_{l \neq k} \mid \phi_{k}\right\rangle \equiv B_{k}^{l} ; \quad\left|B_{k}^{l}\right| \geq 0 .
$$

At the crossing point

$$
A_{k}^{(\mathrm{cr})} \rightarrow \infty \quad\left|B_{k}^{l(\mathrm{cr})}\right| \rightarrow \infty,
$$

for details see [7].

The relation between the eigenfunctions $\phi_{1}$ and $\phi_{2}$ of the operator (21) at the crossing point is

$$
\phi_{1}^{\mathrm{cr}} \rightarrow \pm i \phi_{2}^{\mathrm{cr}} \quad \phi_{2}^{\mathrm{cr}} \rightarrow \mp i \phi_{1}^{\mathrm{cr}}
$$

according to analytical $[17,26]$ as well as numerical studies [27]. The two eigenfunctions are linearly dependent of one another at the crossing point such that the number of eigenfunctions of $H$ is reduced at this point. This result shows once more that the crossing point is an exceptional point in the sense defined by Kato [14].

In an experimental study on a microwave cavity [20], the topological structure of the exceptional point and its surrounding is studied by encircling it and tracing the relative amplitudes of the wavefunctions (field distributions inside the cavity). As a result, the wavefunctions including their phases are restored after four surroundings. The authors [20] interpreted the experimental data by two theoretical assumptions: (i) the two wavefunctions coalesce into one at the exceptional point, $\phi_{1}^{\text {cr }} \leftrightarrow \phi_{2}^{\text {cr }}$, and (ii) only one of the wavefunctions picks up a phase of $\pi$ (a sign change) when encircling the critical point. With these two assumptions, the wavefunctions are restored after four surroundings as found experimentally. 


\section{EPJ Web of Conferences}

The experimental result can be explained, however, without any additional assumptions by using the relations (30)

$$
\begin{array}{rlrl}
\text { 1. cycle : } & \varepsilon_{1,2} \rightarrow \varepsilon_{2,1} & \phi_{1,2} & \rightarrow \pm i \phi_{2,1} \\
\text { 2. cycle : } & \varepsilon_{2,1} \rightarrow \varepsilon_{1,2} & \pm i \phi_{2,1} \rightarrow-\phi_{1,2} \\
\text { 3. cycle : } & \varepsilon_{1,2} \rightarrow \varepsilon_{2,1} & -\phi_{1,2} \rightarrow \mp i \phi_{2,1} \\
\text { 4. cycle : } & \varepsilon_{2,1} \rightarrow \varepsilon_{1,2} & \mp i \phi_{2,1} \rightarrow \phi_{1,2}
\end{array}
$$

As can be seen, the eigenvalues are restored after two surroundings and the eigenfunctions are restored after four surroundings, in full agreement with the experimental result.

In any case, $\left|\phi_{1}^{\mathrm{cr}}\right|=\left|\phi_{2}^{\mathrm{cr}}\right|$ at the crossing point in agreement with the statement that the number of eigenstates is reduced at the exceptional point. The topological phase is twice the Berry phase, in accordance with the enlarged function space in open quantum systems.

Theoretical studies [26] have shown that associated vectors $\phi_{i}^{\text {cra }}$ defined by the Jordan relations, appear at the crossing points. The corresponding equations are

$$
\left(H-\varepsilon_{0}\right) \phi_{1,2}^{\mathrm{cr}}=0 \quad ; \quad\left(H-\varepsilon_{0}\right) \phi_{1,2}^{\mathrm{cra}}=\phi_{1,2}^{\mathrm{cr}} .
$$

The existence of two states in the very neighborhood of the exceptional point has been seen in a numerical calculation for the elastic scattering of a proton on a light nucleus [28]. The elastic scattering phase shifts jump always by $2 \pi$ (and not by $\pi$ as for a single resonance state).

Furthermore, the phases of the wavefunctions jump by $\pi / 4$ at the crossing point (when traced as a function of a parameter) due to the biorthogonality (26) of the eigenfunctions of the non-Hermitian Hamiltonian $H$, see also (28). This result has been proven in many numerical studies, see [7].

Let us now consider the consequences of the biorthogonality relations (26) and (27) for the two borderline cases characteristic of neighboring resonance states.

1. The two levels are distant from one another. Then the eigenfunctions are (almost) orthogonal, $\operatorname{Im}\left(\phi_{k}\right)^{2}$ is small and

$$
\left\langle\phi_{k}^{*} \mid \phi_{k}\right\rangle \approx\left\langle\phi_{k} \mid \phi_{k}\right\rangle=A_{k} \approx 1
$$

2. The two levels cross. Then the two eigenfunctions are linearly dependent according to (30), $\operatorname{Im}\left(\phi_{k}\right)^{2}$ is large and, according (29),

$$
\left\langle\phi_{k}^{*} \mid \phi_{k}\right\rangle=1 \neq\left\langle\phi_{k} \mid \phi_{k}\right\rangle=A_{k} \rightarrow \infty .
$$

The two relations (33) and (34) show that the phases of the two eigenfunctions relative to one another change when the crossing point is approached. This can be expressed quantitatively by defining the phase rigidity $r_{k}$ of the eigenfunctions $\phi_{k}$,

$$
r_{k} \equiv \frac{\left\langle\phi_{k}^{*} \mid \phi_{k}\right\rangle}{\left\langle\phi_{k} \mid \phi_{k}\right\rangle}=A_{k}^{-1}
$$

According to (33) and (34) holds

$$
1 \geq r_{k} \geq 0
$$

The non-rigidity of the phases of the eigenfunctions of $H$ follows also from the fact that $\left\langle\phi_{k}^{*} \mid \phi_{k}\right\rangle$ is a complex number (in difference to the norm $\left\langle\phi_{k} \mid \phi_{k}\right\rangle$ which is a real number) such that the normalization condition (26) can be fulfilled only by the additional postulation $\operatorname{Im}\left\langle\phi_{k}^{*} \mid \phi_{k}\right\rangle=0$ (what corresponds to a rotation [7]).

It should be remarked here that discrete states never cross but always avoid crossing, see section 3.2. The eigenfunctions are real and normalized as $\left\langle\phi_{k} \mid \phi_{k}\right\rangle=1$. Accordingly, $r_{k}=1$ for discrete states also in the region of avoided level crossings. The corresponding exceptional points can be found by analytical continuation into the continuum [17]. 


\section{$\mathrm{CNR} * 11$}

Under the influence of an avoided level crossing, the wavefunctions of the two states are mixed in a certain range of the parameter considered. This range shrinks to one point in the case of a true crossing (exceptional point) but is finite when the levels avoid crossing. It is particularly large for discrete states [17].

The variation of $r_{k}$ according to (36) in approaching the crossing point of two eigenvalue trajectories of resonance states is proven experimentally by means of a study on a microwave cavity [29]. As a result of the experimental study, the phase difference between two modes is $\pi$ at large distance and decreases to $\pi / 2$ at the crossing point.

The authors of [29] interpreted the experimental data by assuming (i) that the singular point is a chiral state (in spite of the phase jump occurring at the crossing point, when traced as a function of a certain parameter), (ii) that the number of states is reduced from 2 to 1 at the crossing point (in spite of the existence of the associate vector (32)) and (iii) that a single point in the continuum can be identified (although it is of measure zero). The authors are unable to explain the large parameter range in which the phase difference decreases in approaching the crossing point.

Considering the phase rigidity $r_{k}$ in the regime of the two overlapping resonance states, the experimental results given in [29], can be explained immediately since the phase rigidity varies smoothly in a comparably large parameter range. It can therefore be concluded that the experimental results [29] prove the statement that the phases of the eigenfunctions of the non-Hermitian Hamilton operator $H$ are not rigid in approaching the crossing point, but vary according to (33) to (36).

The Schrödinger equation with the non-Hermitian operator (21) can be rewritten into a nonlinear Schrödinger equation with Hermitian operator and a source term arising from the coupling to the continuum [7,17]. It goes over smoothly into a linear Schrödinger equation when $A_{k} \equiv\left|\phi_{k}\right|^{2} \rightarrow 1$.

In any case, exceptional points cause spectroscopic changes in the system. Most visible effect is that level repulsion passes into width bifurcation. The changes are accompanied by the non-rigidity of the eigenfunctions of the Hamiltonian, and by nonlinear terms in the equations describing the system around an exceptional point.

\section{Biorthogonal wavefunctions, exceptional points, trapped and aligned states in the case of a many-level system}

According to (17), the solutions of (1) inside the system can be represented in a set of biorthogonal wavefunctions $\Phi_{k}$. They can be normalized according to

$$
\left\langle\Psi_{c \text { int }}^{E, L} \mid \Psi_{c \text { int }}^{E, R}\right\rangle=\sum_{k=1}^{N}\left(c_{k E}^{c}\right)^{2} \rightarrow 1
$$

under the condition that $\operatorname{Re}\left(c_{k E}^{c}\right) \operatorname{Im}\left(c_{k E}^{c}\right)=0$. This corresponds to a rotation as discussed in section 3.3 for the two-level case. It is possible therefore to define the phase rigidity $\rho$ of the $\Psi_{c \text { int }}^{E}$ in the many-level case in analogy to the phase rigidity $r_{k}$ of the $\phi_{k}$ in the two-level case, equation (35). The analogy leads to

$$
\rho=\left|\frac{\int d r \Psi_{c \text { int }}^{E}(r)^{2}}{\int d r\left|\Psi_{c \text { int }}^{E}(r)\right|^{2}}\right|=\left|\frac{\int d r\left(\left[\operatorname{Re} \Psi_{c \text { int }}^{E}\right]^{2}-\left[\operatorname{Im} \Psi_{c \text { int }}^{E}\right]^{2}\right)}{\int d r\left(\left[\operatorname{Re} \Psi_{c \text { int }}^{E}\right]^{2}+\left[\operatorname{Im} \Psi_{c \text { int }}^{E}\right]^{2}\right)}\right|
$$

with

$$
1 \geq \rho \geq 0 .
$$

The phase rigidity $\rho$ describes the internal impurity of the open quantum system which is caused by exceptional points, for details see [7]. It is characteristic of open quantum systems the properties of which are influenced by the coupling to the environment.

Mathematically, the influence of the environment onto the system is described by the non-rigid phases of the wavefunctions $\Psi_{c \text { int }}^{E}$ in the regime of high level density. Under the influence of the 


\section{EPJ Web of Conferences}

environment, width bifurcation occurs and causes spectroscopic redistribution processes in the system: some of the states align to the (extended) scattering states $\xi_{c}^{E}$ of the environment and become shortlived while other ones are trapped and become long-lived.

The spectroscopic redistribution takes place step by step. Due to $1>\rho>0$ in approaching a region with many exceptional points, one (or a few) of the states can finally align with the scattering state $\xi_{c}^{E}$ of the environment. Full alignment is reached for $\rho=0$, i.e. when the spectroscopic overlap between the wavefunction $\Phi_{c \text { int }}^{E}$ with the wavefunction $\xi_{c}^{E}$ is maximal. In any case, the alignment of a few states $\Psi_{c \text { int }}^{E}$ with the channel wavefunctions $\xi_{c}^{E}$ occurs by trapping other states, i.e. by almost complete decoupling them from the environment. The widths of these trapped states are small and decrease with increasing coupling strength between system and environment. Under certain conditions (spatial symmetry), a few of them may even vanish and become bound states in the continuum, see e.g. $[22,23,7]$. The resonance trapping phenomenon is proven experimentally [30].

For discrete states (outside the window $E_{\text {thr }}^{l} \leq E \leq E_{\text {thr }}^{h}$ ) it is always $\rho=1$. Nevertheless, spectroscopic redistribution processes will take place at high level density also in this case due to the principal value integral in (9). The corresponding avoided crossings of the discrete states can be seen and the exceptional points can be found by analytical continuation into the continuum [17].

Numerical studies have shown that the wavefunctions of the two crossing states are mixed in a certain finite range $\Delta a$ of the parameter $a$ around the critical point $a^{\text {cr }}$ [17]. At high level density, the different $\Delta_{i} a$ (related to the crossings of different neighboring levels) overlap. Due to this overlap, spectroscopic redistribution processes occur really in the system [17].

The alignment of resonance states to the scattering states of the environment (by trapping other states) as well as the spectroscopic redistribution of discrete states are caused by avoided level crossings and are a collective phenomenon to which all states in a large energy region contribute, see the following section 5. The principal value integral in (9) does not induce residual two-body forces and one can not expect that the trapped states are describable as individual states with account of only two-body residual forces.

The phenomenon of alignment of resonance states is equivalent to a dynamical phase transition that takes place in a realistic many-level quantum system under the influence of the environment. It is accompanied by the counterintuitive resonance trapping phenomenon.

\section{Dynamical phase transitions under the influence of the environment}

Some years ago, the question has been studied [31] whether or not the resonance trapping phenomenon is related to some type of phase transition. The study is performed by using the toy model

$$
H_{\mathrm{eff}}^{\text {toy }}=H_{0}+i \alpha V V^{+}
$$

in the one-channel case and with the assumption that (almost) all exceptional points accumulate in one point [19]. It has been found that resonance trapping may be understood, in this case, as a secondorder phase transition. The calculations are performed for a linear chain consisting of a finite number $m=2 n+1$ of states. The state in the center of the spectrum traps the other ones and becomes a collective state in a global sense: it contains components of almost all basic states of the system, also of those which are not overlapped by it. This agrees with the appearance of the principal value integral in (9). The normalized width $\Gamma_{0} / m$ of this state can be considered as the order parameter: it increases linearly as a function of $\alpha$, and the first derivative of $\Gamma_{0} / m$ jumps at the critical value $\alpha=\alpha^{\mathrm{cr}}$. The two phases differ by the number of localized states. In the case considered, this number is $m$ at $\alpha<\alpha^{\mathrm{cr}}$, and $m-1$ at $\alpha>\alpha^{\mathrm{cr}}$.

Much more interesting is the realistic case with the Hamiltonian (7). Here, trapping of resonance states occurs in the regime of overlapping resonances hierarchically, i.e. one by one [32]. The crossing points do not accumulate in one point, but are distributed over a certain finite range of the parameter. In this case, a dynamical phase transition takes place in a finite parameter range inside the regime of overlapping resonances [7]. Also in this case, almost all resonance states are involved in the phase transition and, furthermore, the number $N$ of localized states is reduced. That means, the $Q$ subspace 


\section{$\mathrm{CNR} * 11$}

splits into two parts under the influence of the environment. One part contains the few short-lived states which are (more or less) aligned to the scattering states of the environment, while the other part embodies the trapped, long-lived and well localized states. Both time scales are well separated from one another.

An example are the short-lived whispering gallery modes in a microwave cavity with convex boundary which coexist with many long-lived states [33,34]. Another example is known in nuclear physics: the short-lived single-particle resonances are responsible for the fast direct reaction part, while the long-lived ones cause the slow compound-nucleus reaction part. In the Feshbach unified theory of nuclear reactions [12], the direct reaction part is described exactly while the compound-nucleus reaction part is described by means of statistical ensembles. Similar representations are used in other fields of physics at high level density.

Interesting is the enhancement of observable values in the parameter range in which the phase transition takes place [34]. The enhancement is a direct consequence of the alignment that occurs in such a manner that the aligned state fits best to the environment, i.e. that the corresponding $\gamma_{k}^{c}$ is maximal.

It remains the question whether or not the transition regime between the two phases where an enhancement of observable values is expected, can be observed experimentally. The answer to this question depends on the density of exceptional points.

1. When all exceptional points accumulate in one point, the transition regime shrinks to one point in the parameter space. In such a case, a new threshold opens at this point, and the transition regime can not be observed.

2. When the exceptional points are distributed over a finite parameter range, the transition region is observable. In this case, $\rho<1$ in a finite parameter range below the new threshold, that may open when one of the states is maximal aligned. The parameter range is the larger the larger the range with $\rho<1$ is. Accordingly, the transmission through the system is enhanced in a finite parameter region below this new threshold. An example are whispering gallery modes which cause an enhanced transmission through the system when the leads are attached to the cavity in an adequate manner [34]. Moreover, the results of numerical studies show the correlated behavior of conductance and phase rigidity in the transition from the weak-coupling to the strong-coupling regime.

In any case, the regime at low level density (or small coupling via the continuum) differs from the regime at high level density (corresponding to strong coupling via the continuum). At small coupling via the continuum, the resonance states show individual spectroscopic features which are lost at large coupling. Here, many narrow (trapped) resonances are superimposed on broad (aligned) resonances. According to a shot-noise analysis, the trapped resonance states show chaotic features [33].

The dynamical phase transition is surely one of the most interesting features of non-Hermitian quantum physics. It is environmentally induced and appears in systems with and without dissipation. The corresponding alignment is a collective phenomenon to which all resonance states in a large energy region contribute. Mathematically, this phenomenon is directly related to the existence of exceptional points, i.e. to the coupling matrix elements $\omega$ in (21), to the phase rigidity $r_{k}$ of the eigenfunctions and to the nonlinear terms in the Schrödinger equation, see section 3.3.

It should be mentioned here that dynamical phase transitions seem to be similar to the superradiance phase transitions discussed in, e.g., [35-37]. The difference between these two theoretical types of phase transitions consists in the approximations involved in the calculations. In the case of a dynamical phase transition, the wavefunctions $\Phi_{k}^{B}$ of the basic discrete states are calculated explicitly (see section 2). Thus, the interplay between internal and external degrees of freedom is taken into account in a straightforward manner. An example are whispering gallery modes in microwave cavities which appear when the leads are attached to the cavity in a suitable manner [34]. In the study of superradiant phase transitions, however, the coupling term between system and environment is assumed to be purely imaginary and factorizable as in the toy model (40) and the discrete states are assumed mostly to be states of a random ensemble. That means, the internal degrees of freedom of the system are frozen and only the external degrees of freedom (coupling to the continuum) are varied. Furthermore, in such a case, the exceptional points are accumulated in a very small parameter range [31], such that the phase transition shrinks in parameter space to a very small region just below the threshold. 


\section{Dynamical phase transitions in experimental data}

The appearance of dynamical phase transitions can explain some puzzles that are observed experimentally and could not explained theoretically in the framework of conventional Hermitian quantum theory. Some experimental results of such a type will be sketched in the following.

\subsection{Experimental verification of the resonance trapping phenomenon}

First, the experimental verification of the resonance trapping phenomenon will be mentioned here which is performed more than 10 years ago [30]. The experiment is carried out on a microwave cavity to which a waveguide with variable slit is attached. In experiments of this type, the equivalence of the electromagnetic spectrum for a flat cavity to the spectrum of the corresponding quantum mechanical system is used. This equivalence allows to receive, generally, clear results for quantum systems.

The result of this experiment is the following. The widths of all resonance states first increase with increasing coupling strength to the continuum (increasing opening of the slit), but finally decrease again, for most states. They verify therefore clearly the counterintuitive resonance trapping phenomenon.

\subsection{Phase lapses}

The transmission amplitude $|T| e^{i \beta}$ through a many-electron quantum dot is studied experimentally more than 10 years ago in an Aharonov-Bohm ring. The results obtained were surprisingly: $\beta$ jumps sharply downwards by $\pi$ in each valley between any two successive peaks [38]. Only few-electron dots show the expected mesoscopic behavior [39].

These results are a challenge for theoreticians. Many papers appeared in order to explain the results by using conventional Hermitian quantum physics. However, the problem could not be solved convincingly, see e.g. the corresponding Focus Issue of New Journal of Physics 9 (2007).

On the basis of the generic non-Hermitian operator (40), it was possible to explain qualitatively the experimental results [40]. The phase lapses can be considered therefore to verify experimentally the phenomenon of a dynamical phase transition. More concrete calculations will be performed.

\subsection{Quantum dynamical phase transition in the spin swapping operation}

The spin swapping operation is studied experimentally as well as theoretically in several papers [41]. Here, by turning on and off the spin-spin interaction, a splitting of the energy levels occurs and an oscillation with frequency $\omega$ is induced. The interaction $\hbar / \tau_{S E}$ with the environment of neighboring spins degrades oscillation within the decoherence time scale $\tau_{\phi}$.

The experimental (and theoretical) results are the following. It is $1 / \tau_{\phi} \propto 1 / \tau_{S E}$ at low $\tau_{S E}$ (Fermi golden rule) and $1 / \tau_{\phi} \propto \tau_{S E}$ at large $\tau_{S E}$. These results show directly the occurrence of a dynamical phase transition. They are related to the existence of exceptional points in [42].

\subsection{Loss induced optical transparency in complex optical potentials}

The optical wave equation is formally equivalent to the quantum mechanical Schrödinger equation, and PT symmetric optical lattices show, qualitatively, a similar behavior as open quantum systems. The experimental studies in specially designed non-Hermitian guiding potentials show a phase transition which is called, in these studies, PT symmetry breaking. It consists in loss induced optical transparency: the output transmission first decreases, attains a minimum and then increases with increasing loss. [43].

Concrete theoretical studies [44] show the following results. At the phase transition in PT symmetric complex periodic potentials, the modes are skewed (nonorthogonal) and nonreciprocal, expressing 


\section{CNR*11}

the influence of exceptional points. A detailed discussion of this analogy is given in [45]. Thus, also these experimental data show features characteristic of a dynamical phase transition related to exceptional points.

\subsection{Saturation of unimolecular reaction rates in the regime of overlapping resonances}

Many years ago, it has been shown that decay rates saturate at high level density such that the basic relation between decay rates and decay widths seems to be violated [46]. According to the counterintuitive resonance trapping phenomenon, the definition of an average width at high level density is, however, meaningless when summed over all resonance states. The summation has to be restricted to the number of long-lived trapped states. In such a case, also the average decay width saturates. Thus, both the decay rate and the average width saturate at high level density as a function of the averaged total coupling strength to the environment[47]. It is this saturation due to which the optical model is applicable also at very high level density and the condition $\tilde{\Gamma}_{\text {av }} \ll D$ is always fulfilled.

\subsection{Relation between compound nucleus and shell model states}

The main problem of present nuclear physics studies is that there is no parameter by means of which it is possible to control the behavior of the nucleus as a function of a certain parameter. We have therefore only 'snap-shots', and different interpretations of the experimental data are often possible.

Problems are known to appear when trying to relate the compound nucleus states (characteristic of the regime at high level density) to the shell model states (describing well the individual states at low level density). In order to describe the compound nucleus states, the GOE is used mostly. However, the GOE contains many-body forces, shows collective features and is not related to a two-body random ensemble [9]. Moreover, the states of the GOE can not be considered to be independent from one another, since they do not decay according to an exponential law although they are well separated in energy from one another [10]. The compound nucleus states reflect therefore properties characteristic of trapped resonance states (see sections 4 and 5).

This interpretation is supported by some experimental data. Two examples are the following. (i) According to lifetime measurements [4] by using the crystal blocking technique, the average lifetime of the compound nucleus states of some $N i$ isotopes increases with increasing excitation energy and is much larger than expected. Furthermore, signatures for the formation of a comparatively simple configuration are found. (ii) A careful analysis of recent data has shown that the reduced neutron widths of compound nucleus states do not follow a Porter-Thomas distribution [8].

It can be drawn therefore the following conclusion. The compound nucleus states at high level density can be identified with trapped resonance states. They have lost their individual spectroscopic features in a dynamical phase transition and are described best by an ensemble of states. The corresponding aligned states are the broad neutron resonances lying just below the threshold for neutron decay. The dynamical phase transition takes place below the first neutron decay threshold, where the states are discrete (as long as the coupling to photon channels is not considered).

According to this picture, the compound nucleus states have some counterintuitive properties which result from the spectroscopic redistribution processes taking place due to avoided level crossings (caused by exceptional points). Furthermore, the trapped resonance states show chaotic features according to a shot-noise analysis in small quantum cavities with large opening and formation of whispering gallery modes [33].

In contrast to these global properties of the compound nucleus states, the shell model states are characterized by individual spectroscopic features described well by means of the shell model with inclusion of two-body residual forces. This picture of shell model and compound nucleus states agrees fully with the statement given by Niels Bohr in 1936 [1]. 


\section{EPJ Web of Conferences}

\section{Summary}

In the present paper, the nucleus is described as an open quantum system with a non-Hermitian Hamilton operator by using the Feshbach projection operator formalism. Of special interest is the regime at high level density where exceptional points, avoided level crossings of eigenvalue trajectories, width bifurcation and reduced phase rigidity of the eigenfunctions play an important role. In the regime of overlapping resonances, a few resonance states align to the scattering states of the environment by trapping (decoupling from the environment) the other states. Analog spectroscopic redistribution processes take place below the particle decay threshold where the states are discrete. In this case, the corresponding exceptional points can be found by analytical continuation into the continuum. Due to the spectroscopic redistribution, all states lose their spectroscopic relation to the individual states at low level density, and a dynamical phase transition takes place. The aligned states are short-lived while the trapped ones are long-lived.

The alignment of resonance states (and the accompanying resonance trapping) is a collective phenomenon to which all states in a large energy region contribute. It is therefore a global phenomenon (and not a local one) which is environmentally induced. Mathematically, the resonance trapping phenomenon is caused by the existence of many exceptional points in the regime of high level density. Correspondingly, the number of long-lived (trapped) resonance states is reduced while a few shortlived (aligned) states are formed.

Dynamical phase transitions are observed experimentally in different small quantum systems and in systems equivalent to them. They are characteristic of non-Hermitian quantum physics at high level density. Many of the results are counterintuitive when considered from the point of view of Hermitian quantum physics. For example, the Fermi golden rule is violated under certain conditions (section 6.3) or unexpected regularities appear in the spectra (section 6.2).

Dynamical phase transitions appear also in nuclei. While the nuclear states at low level density are described well by the shell model with account of two-body residual forces, many properties of the compound nucleus states are described well by e.g. the GOE. It contains the global (collective) features characteristic of trapped resonance states. The states are members of an ensemble and different from states with individual spectroscopic features (in spite of the fact that they appear well isolated from one another in energy). Furthermore, they show some counterintuitive features characteristic of trapped states, e.g. an increase of the average lifetime with increasing energy.

As a conclusion, the state of the art is the following. Dynamical phase transitions taking place at high level density, prove Bohr's statement [1]: the properties of low-lying nuclear states (described by the shell model) and those of highly excited nuclear states (described by random ensembles) differ fundamentally from one another.

\section{References}

1. N. Bohr, Nature 137 (1936) 344; Naturwissenschaften 24 (1936) 241.

2. The Nobel Prize in Physics 1963: Maria Goeppert-Mayer and J. Hans D. Jensen for their discoveries concerning nuclear shell structure.

3. J.P. Jeukenne and C. Mahaux, Nucl.Phys.A 136 (1969) 49

4. E.P. Kanter, D. Kollewe, K. Komaki, I. Leuca, G.M. Temmer and W.M. Gibson, Nucl. Phys. A 299 (1978) 230

5. G.M. Temmer, private communication (around 1985)

6. I. Rotter, Rep. Prog. Phys. 54 (1991) 635

7. I. Rotter, J. Phys. A 42 (2009) 153001, and references therein

8. P.E. Koehler, F. Becvar, M. Krticka, J.A. Harvey, and K.H. Guber, Phys. Rev. Lett. 105 (2010) 072502

9. T. Guhr, private communication (2010)

10. F.M. Dittes, H.L. Harney and A. Müller, Phys. Rev. A 45 (1992) 701; H.L. Harney, F.M. Dittes and A. Müller, Ann. Phys. (NY) 220 (1992) 159

11. S. Drozdz, J. Okolowicz, M. Ploszajczak, and I. Rotter, Phys. Rev. C 62 (2000) 024313 


\section{$\mathrm{CNR} * 11$}

12. H. Feshbach, Ann. Phys. (NY) 5 (1958) 357 and Ann. Phys. (NY) 19 (1962) 287

13. V.V. Shamshutdinova, K.N. Pichugin, I. Rotter, B.F. Samsonov, Phys. Rev. A 78 (2008) 062712

14. T. Kato, Peturbation Theory for Linear Operators, Springer Berlin, 1966

15. L. Landau, Physics Soviet Union 2 (1932) 46; C. Zener, Proc. Royal Soc. London, Series A 137 (1932) 692.

16. M.V. Berry, Proc. R. Soc. London, Ser. A 392 (1984) 45; M.V. Berry and M. Wilkinson, Proc. R. Soc. London, Ser. A 392 (1984) 15

17. I. Rotter, Phys. Rev. E 64 (2001) 036213

18. E. Persson, K. Pichugin, I. Rotter, and P. Seba, Phys. Rev. E 58 (1998) 8001

19. W.D. Heiss, M. Müller, and I. Rotter, Phys. Rev. E 58 (1998) 2894

20. C. Dembowski, H.-D. Gräf, H. L. Harney, A. Heine, W.D. Heiss, H. Rehfeld, and A. Richter, Phys. Rev. Lett. 86 (2001) 787

21. H. Friedrich and D. Wintgen, Phys. Rev. A 31 (1985) 3964 (1985) and 32 (1985) 3231

22. A.I. Magunov, I. Rotter and S.I. Strakhova, J. Phys. B 32 (1999) 1669

23. I. Rotter and A.F. Sadreev, Phys. Rev. E 71 (2005) 046204

24. A. Mostafazadeh, Phys. Rev. Lett. 102 (2009) 220402

25. S. Longhi, Phys. Rev. B 80 (2009) 165125

26. U. Günther, I. Rotter and B.F. Samsonov, J. Phys. A 40 (2007) 8815

27. A.I. Magunov, I. Rotter and S.I. Strakhova, J. Phys. B 34 (2001) 29

28. J. Okolowicz and M. Ploscajczak, Phys. Rev. C 80 (2009) 034619

29. C. Dembowski, B. Dietz, H.D. Gräf, H.L. Harney, A. Heine, W.D. Heiss, and A. Richter, Phys. Rev. Lett. 90 (2003) 034101

30. E. Persson, I. Rotter, H.J. Stöckmann, and M. Barth, Phys. Rev. Lett. 85 (2000) 2478

31. C. Jung, M. Müller, and I. Rotter, Phys. Rev. E 60 (1999) 114

32. W. Iskra, I. Rotter, and F.M. Dittes, Phys. Rev. C 47 (1993) 1086

33. R.G. Nazmitdinov, H.S. Sim, H. Schomerus and I. Rotter, Phys. Rev. B 66 (2002) 241302(R)

34. E.N. Bulgakov, I. Rotter and A.F. Sadreev, Phys. Rev. E 74 (2006) 056204 and Phys. Rev. B 76 (2007) 214302

35. V.V. Sokolov and V.G. Zelevinsky, Ann. Phys. (N.Y.) 216 (1992) 323

36. G.L. Celardo and L. Kaplan, Phys. Rev. B 79, (2009) 155108; G.L. Celardo, N. Auerbach, F.M. Izrailev, and V.G. Zelevinsky, Phys. Rev. Lett. 106 (2011) 042501

37. A. Volya, Phys. Rev. C 83 (2011) 044312

38. A. Yacoby, M. Heiblum, D. Mahalu and H. Shtrikman, Phys. Rev. Lett. 74 (1995) 4047; R. Schuster, E. Buks, M. Heiblum, D. Mahalu, V. Umansky and H. Shtrikman, Nature (London) 385 (1997) 417

39. M. Avinun-Kalish, M. Heiblum, O. Zarchin, D. Mahalu and V. Umansky, Nature (London) 436 (2005) 529

40. M. Müller and I. Rotter, Phys. Rev. A 80 (2009) 042705

41. G.A. Alvarez, E.P. Danieli, P.R. Levstein, H.M. Pastawski, J.Chem.Phys. 124 (2006) 194507; H.M. Pastawski, Physica B 398 (2007) 278

42. A.D. Dente, R.A. Bustos-Marun and H.M. Pastawski, Phys. Rev. A 78 (2008) 062116

43. A. Guo, G.J. Salamo, D. Duchesne, R. Morandotti, M. Volatier-Ravat, V. Aimez, G.A. Siviloglou and D.N. Christodoulides, Phys. Rev. Lett. 103 (2009) 093902; C.E. Rüter, K.G. Makris, R. ElGanainy, D.N. Christodoulides, M. Segev and D. Kip, Nature Physics 6, 192 (2010); T. Kottos, Nature Physics 6 (2010) 166

44. K.G. Makris, R. El-Ganainy, D.N. Christodoulides, and Z.H. Musslimani, Phys. Rev. A 81 (2010) 063807

45. I. Rotter, J. Opt. 12 (2010) 065701

46. U. Peskin, H. Reisler, W.H. Miller, J. Chem. Phys. 101 (1994) 9672

47. I. Rotter, J. Chem. Phys. 106 (1997) 4810; U. Peskin, H. Reisler, W.H. Miller, J. Chem. Phys. 106 (1997) 4812 